\title{
DIRECT METHOD OF RESEARCH OF THE TEMPERATURE FIELD IN THE SYSTEM OF MULTILAYER SPHERICAL SHELL
}

\author{
Roman Tatsii; Oleg Pazen
}

\author{
Lviv State University of Life Safety, Lviv, Ukraine
}

\begin{abstract}
Summary. The article is devoted to the application of the direct method to the research of heat transfer processes in the system - bullet inside multilayered spherical shell. To solve the initial problem in parallel, we put an auxiliary problem on the determination of the distribution of a unsteady temperature field in a multilayer spherical solid with a «deleted» bullet of sufficiently small radius. Implementation of the solution of the auxiliary problem is carried out by applying the reduction method using the concept of quasi derivatives. In the future, is used the Fourier scheme with the use of the modified eigenfunctions method. To find the solution of the original problem used the idea of the marginal transition by direction of the radius of the deleted bullet to zero. It is established that in this approach all the eigenfunctions of the corresponding problem with the eigenvalues have no singularities at zero, which means that the solutions of the original problem are limited in the whole construction.
\end{abstract}

Key words: multilayer sphere, quasi derivative, direct method, marginal transition.

Statement of the problem. Multilayer structures are widely used in various industries: civil engineering (house-sphere), oil and gas industry (spherical reservoirs or gas holders), aerospace (artificial Earth satellites), electric power (fuel elements of spherical form in the nuclear power plant reactors) and other fields. The characteristic feature of such elements is the combination of various mechanical and thermophysical layers characteristics, which makes them more perfect. However, this approach poses significant difficulties in developing analytical methods for their investigation. Therefore, the development of new methods for investigating the multilayer, particularly, spherical structures is an important task of the present time.

Analysis of the available investigations results. Numerous publications deals with the heat transfer problem solution. The basic methods of investigating the problems of determining the non-stationary temperature field distribution in the multilayer structures are conventionally divided into three types: a) direct or classical based on the variables separation method [1, 2]; b) operational using various kinds of integral transformations [3]; c) approximated analytical and numerical methods $[4,5]$. Thus, in papers [1,2], the temperature field distribution in the multilayer hollow spherical structure is investigated, taking into account the boundary conditions, which, however, do not depend on time. For the first time the problem of determining the temperature field distribution in the «sphere enclosed in the single-layer spherical shell» system located in the medium with zero temperature was set in paper [3] by A. V. Lykov. The solution of this problem is based on Laplace transformation method, but the algorithm for solving even the problem in images is not presented, but only the final result is given.

In recent years the multilayer hollow cylindrical and spherical structures are considered in papers $[6,7]$. The basis of these publications is the direct (classical) research scheme based on the method of reduction, the concept of quasi-derivatives, the modern theory of linear differential equations systems, the modified method of Fourier eigenfunctions. 
The objective of the paper is to investigate the non-stationary temperature field distribution in the system - sphere inside multilayer spherical shell (structure) when the conditions for heat exchange with the environment, whose temperature varies over time are determined on the surface.

Statement of the original problem. At the initial moment of time $\tau=0$, the sphere with radius $r=r_{0}$ is inserted into the multilayer hollow construction with radii $r_{0}<r_{1}<r_{2}<\cdots<r_{n-1}<r_{n}$ having the same initial temperature $T=T_{0}$ coinciding with the environment temperature. There is perfect thermal contact between the spheres.

On the outer surface of the multilayer hollow sphere, there is convective heat exchange with the environment, i. e., the third-rate boundary conditions are met. The environment temperature the varies according to certain law $\psi(\tau)$, i. e., depends on time $\tau$. It is necessary to find the non-stationary temperature field $T(r, \tau)$ distribution at any given time $\tau$ in such complex system (Fig. 1).

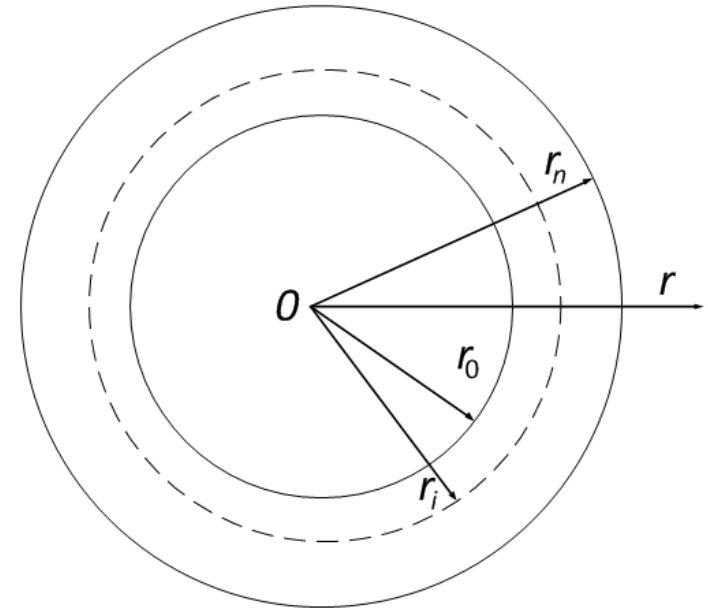

Figure 1. Scheme of the system of multilayered spherical bullet (diametrical section)

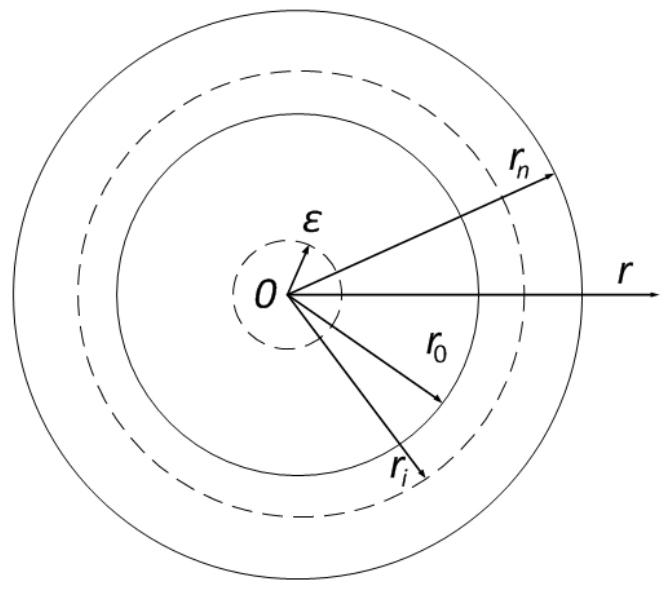

Figure 2. Scheme of multilayer hollow spherical construction

It is considered that the temperature change law $\psi(\tau)$ is uniformly distributed in the external surface layer in such a way that the isotherms within this structure are concentric spheres. This means that the temperature $T(r, \tau)$ depends only on the radius $r$ and time $\tau$ and the problem is symmetric.

Such problem statement is reduced to solving the differential equation of heat conductivity [3]

$$
c \rho \frac{\partial T(r, \tau)}{\partial \tau}=\frac{1}{r^{2}} \frac{\partial}{\partial r}\left(r^{2} \lambda \frac{\partial T(r, \tau)}{\partial r}\right), r \in\left(0, r_{n}\right), \quad \tau>0,
$$

with initial condition

$$
T(r, 0)=T_{0}
$$

boundary condition 


$$
-\lambda \frac{\partial T}{\partial r}\left(r_{n}, \tau\right)=\alpha\left(T\left(r_{n}, \tau\right)-\psi(\tau)\right)
$$

and symmetry condition

$$
\lambda \frac{\partial T}{\partial r}(0, \tau)=0
$$

Here $c(r)$ is mass specific thermal capacity of the material, $\rho(r)$ is material density, $\lambda(r)$ its thermal conductivity coefficient, $T(r, \tau)$ is temperature depending on radius $r$ and time $\tau$.

Auxuliary problem. In the system, the sphere inside the multilayer shell, let us «remove» the sphere with radius $r=\varepsilon, \quad 0<\varepsilon<r_{0}$, and consider mixed heat conduction problem for the multilayer hollow spherical structure presented in Fig. 2. In order to distinguish the temperature field distribution function $T(r, \tau)$ of the original problem from the distribution function of auxiliary problem, we denote it as $t(r, \tau)$.

Let us suppose that $\theta_{i}$ is the characteristic function [8] at intervals $\left[\varepsilon, r_{n}\right)$ i. e.

$$
\theta_{0}(r)=\left\{\begin{array}{l}
1, r \in\left[\varepsilon, r_{0}\right), \\
0, r \notin\left[\varepsilon, r_{0}\right),
\end{array} \quad \theta_{i+1}(r)=\left\{\begin{array}{l}
1, r \in\left[r_{i}, r_{i+1}\right), \\
0, r \notin\left[r_{i}, r_{i+1}\right), i=\overline{0, n-1}
\end{array}\right.\right.
$$

Let us denote, $\lambda(r)=\sum_{i=0}^{n-1} \lambda_{i} \theta_{i}, \quad c(r) \rho(r)=\sum_{i=0}^{n-1} c_{i} \rho_{i} \theta_{i}, \quad \lambda_{i}>0, \quad c_{i} \rho_{i}>0, \quad \forall i=\overline{0, n-1}$, $\lambda_{i}, c_{i}, \rho_{i} \in R$.

In order to find non-stationary temperature field distribution $t(r, \tau)$ in this multilayer hollow spherical structure it is necessary to find the solution of the heat conductivity differential equation [7]

$$
c \rho \frac{\partial t(r, \tau)}{\partial \tau}=\frac{1}{r^{2}} \frac{\partial}{\partial r}\left(r^{2} \lambda \frac{\partial t(r, \tau)}{\partial r}\right), r \in\left(\varepsilon, r_{n}\right), \quad \tau>0,
$$

with initial condition

$$
t(r, 0)=t_{0}
$$

and boundary conditions

$$
\left\{\begin{array}{c}
\lambda \frac{\partial t}{\partial r}(\varepsilon, \tau)=0 \\
-\lambda \frac{\partial t}{\partial r}\left(r_{n}, \tau\right)=\alpha\left(t\left(r_{n}, \tau\right)-\psi(\tau)\right)
\end{array}\right.
$$


Introducing notation $t^{[1]} \stackrel{d f}{=} r^{2} \lambda t_{r}^{\prime}$, and multiplying the boundary conditions (7) by $r^{2}$ we get

$$
\left\{\begin{array}{c}
t^{[1]}(\varepsilon, \tau)=0, \\
\alpha r_{n}^{2} t\left(r_{n}, \tau\right)+t^{[1]}\left(r_{n}, \tau\right)=\alpha r_{n}^{2} \psi(\tau),
\end{array}\right.
$$

The solution of the problem (5)-(6) will be found by means of reduction method $[9,10]$

$$
t(r, \tau)=u(r, \tau)+v(r, \tau)
$$

where one of the functions $(u(r, \tau)$ or $v(r, \tau))$ is chosen in special way, and the other one is defined uniqely.

The boundary problem for $u(r, \tau)$ and mixed problem for $v(r, \tau)$. Let us introduce the following notation $r^{2} \lambda u_{r}^{\prime}=u^{[f]}$. Let us define function $u(r, \tau)$ as the solution of boundary (quasi-stationary) problem:

$$
\left(r^{2} \lambda u^{\prime}\right)_{r}^{\prime}=0
$$

with boundary conditions

$$
\left\{\begin{array}{c}
u^{[1]}(\varepsilon, \tau)=0, \\
\alpha r_{n}^{2} u\left(r_{n}, \tau\right)+u^{[1]}\left(r_{n}, \tau\right)=\alpha r_{n}^{2} \psi(\tau),
\end{array}\right.
$$

Substituting (9) into equation (5), taking into account (10), successively we get mixed problem for function $v(r, \tau)$

$$
\begin{gathered}
c \rho \frac{\partial}{\partial \tau}(u+v)=\frac{1}{r^{2}} \frac{\partial}{\partial r}\left(r^{2} \lambda \frac{\partial}{\partial r}(u+v)\right) \Rightarrow \\
c \rho \frac{\partial u}{\partial \tau}+c \rho \frac{\partial v}{\partial \tau}=\frac{1}{r^{2}} \frac{\partial}{\partial r}\left(r^{2} \lambda \frac{\partial v}{\partial r}\right) \Rightarrow c \rho \frac{\partial v}{\partial \tau}=\frac{1}{r^{2}} \frac{\partial}{\partial r}\left(r^{2} \lambda \frac{\partial v}{\partial r}\right)-c \rho \frac{\partial u}{\partial \tau} .
\end{gathered}
$$

Since the boundary conditions (8) for function $u(r, \tau)$ meet the boundary conditions (7) for function $t(r, \tau)$, we derive zero boundary conditions for determining function $v(r, \tau)$, i. e.,

$$
\left\{\begin{array}{c}
v^{[1]}(\varepsilon, \tau)=0, \\
\alpha r_{n}^{2} v\left(r_{n}, \tau\right)+v^{[1]}\left(r_{n}, \tau\right)=0,
\end{array}\right.
$$

and the initial condition is as follows

$$
v(r, 0)=t_{0}-u(r, 0)=t_{0}
$$




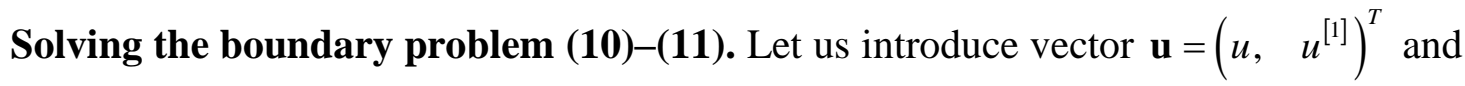
matrix $A=\left(\begin{array}{cc}0 & \frac{1}{r^{2} \lambda} \\ 0 & 0\end{array}\right)$. Then the quasifferential equation (10) is reduced to the solution of the first order differential equations system equivalent to it (11)

$$
\mathbf{u}^{\prime}=A \mathbf{u}
$$

On each interval $\left[\varepsilon, r_{0}\right)$ and $\left[r_{i-1}, r_{i}\right)$ the system (15) is

$$
\mathbf{u}_{0}^{\prime}=A_{0} \mathbf{u}_{0}, \quad A_{0}=\left(\begin{array}{cc}
0 & \frac{1}{r^{2} \lambda_{0}} \\
0 & 0
\end{array}\right), \mathbf{u}_{i}^{\prime}=A_{i} \mathbf{u}_{i}, \quad A_{i}=\left(\begin{array}{cc}
0 & \frac{1}{r^{2} \lambda_{i}} \\
0 & 0
\end{array}\right)
$$

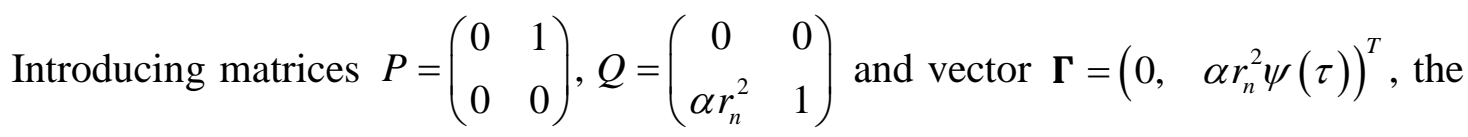
boundary conditions (11) are represented in the matrix

$$
P \cdot \mathbf{u}(\varepsilon)+Q \cdot \mathbf{u}\left(r_{n}\right)=\boldsymbol{\Gamma}
$$

The general equation solution (10) is as follows

$$
u(r)=C_{1}+\frac{C_{2}}{r}
$$

The fundamental system of solutions is formed by functions $\left\{1 ; r^{-1}\right\}$.

The direct verification proves that Cauchy matrix $B_{i}(r, s)$ of the system (16) is

$$
B_{i}(r, s)=\left(\begin{array}{cc}
1 & \frac{1}{\lambda_{i}}\left(\frac{1}{s}-\frac{1}{r}\right) \\
0 & 1
\end{array}\right)
$$

It is determined in paper [11] that on each interval the problem (10)-(11) solution is as follows

$$
\begin{gathered}
\mathbf{u}_{0}(r, \tau)=B_{0}(r, \varepsilon) \cdot \mathbf{P}_{\mathbf{0}}, \\
\mathbf{u}_{i}(r, \tau)=B_{i}\left(r, r_{0}\right) \cdot B\left(r_{i}, r_{0}\right) \cdot B\left(r_{0}, \varepsilon\right) \cdot \mathbf{P}_{\mathbf{0}},
\end{gathered}
$$

where $\mathbf{P}_{\mathbf{0}}$ is the initial vector determined by the following formula: 


$$
\begin{aligned}
& \mathbf{P}_{\mathbf{0}}=\left(P+Q \cdot B\left(r_{n}, \varepsilon\right)\right)^{-1} \cdot \boldsymbol{\Gamma}= \\
& =\left[\left(\begin{array}{ll}
0 & 1 \\
0 & 0
\end{array}\right)+\left(\begin{array}{cc}
0 & 0 \\
\alpha r_{n}^{2} & 1
\end{array}\right) \cdot\left(\begin{array}{cc}
1 & \frac{1}{\lambda_{0}}\left(\frac{1}{\varepsilon}-\frac{1}{r_{0}}\right)+\sum_{i=1}^{n} \frac{1}{\lambda_{i}}\left(\frac{1}{r_{i-1}}-\frac{1}{r_{i}}\right) \\
0 & 1
\end{array}\right)\right]^{-1} \cdot\left(\begin{array}{c}
0 \\
\alpha r_{n}^{2} \psi(\tau)
\end{array}\right)= \\
& =\left(\begin{array}{cc}
0 & 1 \\
\alpha r_{n}^{2} & \alpha r_{n}^{2}\left[\frac{1}{\lambda_{0}}\left(\frac{1}{\varepsilon}-\frac{1}{r_{0}}\right)+\sum_{i=1}^{n} \frac{1}{\lambda_{i}}\left(\frac{1}{r_{i-1}}-\frac{1}{r_{i}}\right)\right]+1
\end{array}\right)^{-1} \cdot\left(\begin{array}{c}
0 \\
r_{n}^{2} \psi(\tau)
\end{array}\right)=
\end{aligned}
$$

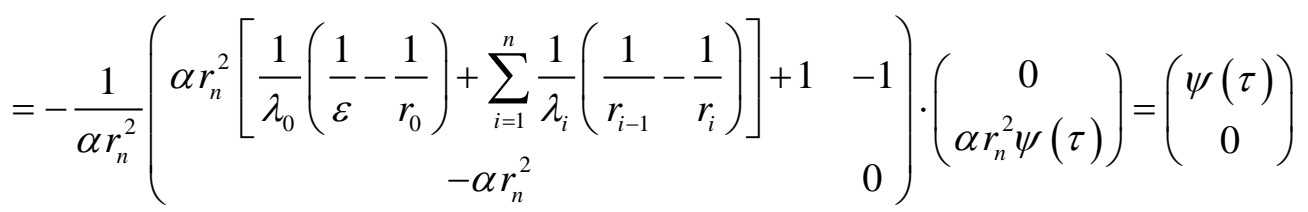

Relatively, the solution of problem (10)-(11) on each interval and $\left[\varepsilon, r_{0}\right)$ and $\left[r_{i-1}, r_{i}\right)$ is represented in the following way:

$$
\begin{aligned}
& \mathbf{u}_{0}(r)=\left(\begin{array}{cc}
1 & \frac{1}{\lambda_{0}}\left(\frac{1}{\varepsilon}-\frac{1}{r}\right) \\
0 & 1
\end{array}\right) \cdot\left(\begin{array}{c}
\psi(\tau) \\
0
\end{array}\right)=\left(\begin{array}{c}
\psi(\tau) \\
0
\end{array}\right) \\
& \mathbf{u}_{i}(r)=\left(\begin{array}{cc}
1 & \frac{1}{\lambda_{i}}\left(\frac{1}{r_{i}}-\frac{1}{r}\right)+\sum_{k=1}^{i-1} \frac{1}{\lambda_{k}}\left(\frac{1}{r_{k-1}}-\frac{1}{r_{k}}\right) \\
0 & 1
\end{array}\right) \cdot\left(\begin{array}{cc}
1 & \frac{1}{\lambda_{0}}\left(\frac{1}{\varepsilon}-\frac{1}{r_{0}}\right) \\
0 & 1
\end{array}\right) \cdot\left(\begin{array}{c}
\psi(\tau) \\
0
\end{array}\right)=\left(\begin{array}{c}
\psi(\tau) \\
0
\end{array}\right)
\end{aligned}
$$

Thus the solution of the boundary problem (10)-(11) is $u(r, t) \equiv \psi(\tau)$, i. e., does not depend on radius $r$.

Solving the mixed inhomogeneous problem for function $v(r, \tau)$. Since function $u(r, \tau)$ is already known, then problem (12) for function $v(r, \tau)$ is

$$
c \rho \frac{\partial v}{\partial \tau}=\frac{1}{r^{2}} \frac{\partial}{\partial r}\left(r^{2} \lambda \frac{\partial v}{\partial r}\right)-c \rho \psi^{\prime}(\tau)
$$

with zero boundary conditions (13) under initial condition (14)

Fourier method and eigenvalue problem. Nontrivial solutions of homogeneous differential equation

$$
c \rho \frac{\partial v}{\partial \tau}=\frac{1}{r^{2}} \frac{\partial}{\partial r}\left(r^{2} \lambda \frac{\partial v}{\partial r}\right)
$$

with boundary conditions (13) we find in the following way [9]

$$
v(r, \tau)=e^{-\omega \tau} \cdot R(r),
$$

where $\omega-$ is parameter, and $R(r)-$ is unknown function. 
Introducing quasiderivative $R^{[1]}=r^{2 f} \lambda R^{\prime}$, and substituting the right part (18) in equation (17) we get quasidifferential equation [7]

$$
\left(r^{2} \lambda R^{\prime}\right)^{\prime}+\omega c \rho r^{2} R=0
$$

with boundary conditions

$$
\left\{\begin{array}{c}
R^{[1]}(\varepsilon)=0 \\
\alpha r_{n}^{2} R\left(r_{n}\right)+R^{[1]}\left(r_{n}\right)=0
\end{array}\right.
$$

Problem (19), (20) is classical problem of eigenvalues where it is necessary to find values $\omega_{k}$ (eigenvalues) for which corresponding nontrivial solutions $R_{k}\left(r, \omega_{k}\right)$ (eigenfunctions) of problem (19), (20) exist. As is known [9], all the eigenvalues of this problem are positive and different.

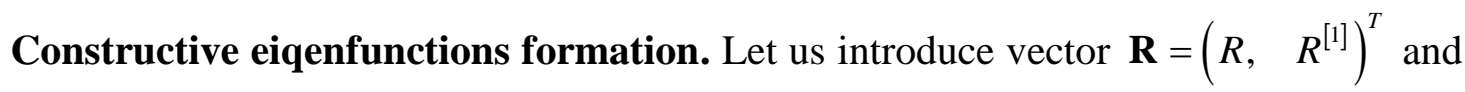
matrix $D(r)=\left(\begin{array}{cc}0 & \frac{1}{r^{2} \lambda(r)} \\ -\omega c(r) \rho(r) r^{2} & 0\end{array}\right)$, reduce quasidifferential equation (19) to equivalent system of differential equations of the first order

$$
\mathbf{R}^{\prime}=D \mathbf{R}
$$

The corresponding system on intervals $\left[\varepsilon, r_{0}\right)$ and $\left[r_{i-1}, r_{i}\right)$ is presented in the following

$$
\begin{gathered}
\mathbf{R}_{0}^{\prime}=D_{0} \cdot \mathbf{R}_{0}, \quad \mathbf{R}_{i}^{\prime}=D_{i} \cdot \mathbf{R}_{i}, \\
D_{0}=\left(\begin{array}{cc}
0 & \frac{1}{r^{2} \lambda_{0}} \\
-\omega c_{0} \rho_{0} r^{2} & 0
\end{array}\right), \quad D_{i}=\left(\begin{array}{cc}
0 & \frac{1}{r^{2} \lambda_{i}} \\
-\omega c_{i} \rho_{i} r^{2} & 0
\end{array}\right) .
\end{gathered}
$$

Equation (19) can be rewritten as

$$
\left(r^{2} R^{\prime}\right)^{\prime}+\beta_{i}^{2} r^{2} R=0
$$

where $\beta_{i}=\sqrt{\frac{\omega c_{i} \rho_{i}}{\lambda_{i}}} \quad i=\overline{0, n-1}$ is denoted.

It is determined in paper [7] that Cauchy matrix of system (21) is as follows

$$
C_{i}(r, s, \omega)=\left(\begin{array}{ll}
b_{11}^{i} & b_{12}^{i} \\
b_{21}^{i} & b_{22}^{i}
\end{array}\right)
$$




$$
\begin{gathered}
b_{11}^{i}=\frac{\beta_{i} s \cos \left(\beta_{i}(r-s)\right)+\sin \left(\beta_{i}(r-s)\right)}{\beta_{i} r} \\
b_{21}^{i}=\frac{\beta_{i} \cos \left(\beta_{i} \cdot(r-s)\right)[r-s]-\sin \left(\beta_{i}(r-s)\right)\left[1+\beta_{i}^{2} r s\right]}{\beta_{i} \beta_{i} r s} \lambda_{i} \\
b_{22}^{i}=\frac{\beta_{i} r \cos \left(\beta_{i}(r-s)\right)-\sin \left(\beta_{i}(r-s)\right)}{\beta_{i} s} \\
i=0,1 .
\end{gathered}
$$

The non-trivial solutions $\mathbf{R}(r, \omega)$ of the system (21) are found in the form

$$
\mathbf{R}(r, \omega)=C(r, \varepsilon, \omega) \cdot \mathbf{C}
$$

$$
C(r, \varepsilon, \omega)=C_{0}(r, \varepsilon, \omega) \theta_{0}+C_{1}\left(r, r_{0}, \omega\right) C_{0}\left(r_{0}, \varepsilon, \omega\right) \theta_{1}+\ldots+
$$
where $+C_{n}\left(r, r_{n-1}, \omega\right) \prod_{i=1}^{n-1} C_{i}\left(r_{i}, r_{i-1}, \omega\right) C_{0}\left(r_{0}, \varepsilon, \omega\right) \theta_{n} \quad$, and $\mathbf{C}=\left(C_{1}, \quad C_{2}\right)^{T}-$ is

certain non-zero vector.

Applying boundary conditions (20) (at $\boldsymbol{\Gamma}(\tau) \equiv 0$ ) to equation (22) we get

$$
P \cdot \mathbf{R}(\varepsilon, \omega)+Q \cdot \mathbf{R}\left(r_{n}, \omega\right)=\left[P \cdot C(\varepsilon, \varepsilon, \omega)+Q \cdot C\left(r_{n}, \varepsilon, \omega\right)\right] \cdot \mathbf{C}=0,
$$

Since $C(\varepsilon, \varepsilon, \omega)=E$, де $E-$ is matrix unit we derive the equation

$$
\left[P+Q \cdot C\left(r_{n}, \varepsilon, \omega\right)\right] \cdot \mathbf{C}=0
$$

For non-trivial vector $\mathbf{C}$ existence in (23) it is necessary and sufficient to meet the following condition

$$
\operatorname{det}\left[P+Q \cdot C\left(r_{n}, \varepsilon, \omega\right)\right]=0
$$

Let us denote

$$
\begin{gathered}
C\left(r_{n}, r_{0}, \omega\right)=C_{n}\left(r_{n}, r_{n-1}, \omega\right) \cdot C_{n-1}\left(r_{n-1}, r_{n-2}, \omega\right) \cdot \ldots \cdot C_{1}\left(r_{1}, r_{0}, \omega\right)= \\
=\left(\begin{array}{ll}
c_{11} & c_{12} \\
c_{21} & c_{22}
\end{array}\right)
\end{gathered}
$$

Equation (24) is the characteristic equation of the problem for eigenvalues (19), (20), which can be expanded to the following form: 


$$
\begin{gathered}
\left(\alpha r_{n}^{2} c_{11}+c_{21}\right) \cdot \frac{\beta_{0} \varepsilon \cos \left(\beta_{0}\left(r_{0}-\varepsilon\right)\right)+\sin \left(\beta_{0}\left(r_{0}-\varepsilon\right)\right)}{\beta_{0} r_{0}}+ \\
+\left(\alpha r_{n}^{2} c_{12}+c_{22}\right) \cdot \frac{\beta_{0} \cos \left(\beta_{0} \cdot\left(r_{0}-\varepsilon\right)\right)\left[r_{0}-\varepsilon\right]-\sin \left(\beta_{0}\left(r_{0}-\varepsilon\right)\right)\left[1+\beta_{0}{ }^{2} r_{0} \varepsilon\right]}{\beta_{0}} \cdot \lambda_{0}=0
\end{gathered}
$$

In order to find non-trivial vector $\mathbf{C}=\left(C_{1}, C_{2}\right)^{T}$ let us introduce $\omega_{k}$ into equality (23) instead of $\omega$. Using notation (25) we get vector equation

$$
\left(\begin{array}{cc}
0 & 1 \\
\left(\alpha r_{n}^{2} c_{11}+c_{21}\right) b_{11}^{0}+\left(\alpha r_{n}^{2} c_{12}+c_{22}\right) b_{21}^{0} & \left(\alpha r_{n}^{2} c_{11}+c_{21}\right) b_{12}^{0}+\left(\alpha r_{n}^{2} c_{12}+c_{22}\right) b_{22}^{0}
\end{array}\right) \cdot\left(\begin{array}{l}
C_{1} \\
C_{2}
\end{array}\right)=\left(\begin{array}{l}
0 \\
0
\end{array}\right),
$$

which is equivalent to the system of equations:

$$
\left\{\begin{array}{l}
C_{2}=0, \\
{\left[\left(\alpha r_{n}^{2} c_{11}+c_{21}\right) b_{11}^{0}+\left(\alpha r_{n}^{2} c_{12}+c_{22}\right) b_{21}^{0}\right] C_{1}+\left[\left(\alpha r_{n}^{2} c_{11}+c_{21}\right) b_{12}^{0}+\left(\alpha r_{n}^{2} c_{12}+c_{22}\right) b_{22}^{0}\right] C_{2}=0 .}
\end{array}\right.
$$

Since the determinant of this system is zero, then one of the equations (for example, the second one) can be neglected. Hence it follows that $C_{2}=0$ for any nonzero value $C_{1}$. Introducing, for example, $C_{1}=1$ we can present non-trivial vector $\mathbf{C}$ as:

$$
\mathbf{C}=(1,0)^{T}
$$

Non-trivial solution corresponding to system (19) is presented in the following way

$$
\mathbf{R}_{k}\left(r, \omega_{k}\right)=\tilde{B}(r, \varepsilon, \omega) \cdot(1, \quad 0)^{T} \stackrel{d f}{=} \mathbf{R}_{k 0} \theta_{0}+\sum_{i=1}^{n-1} \mathbf{R}_{k i} \theta_{i}, \quad k=1,2,3, \ldots
$$

Eigenvectors on intervals $\left[\varepsilon, r_{0}\right)$ and $\left[r_{i-1}, r_{i}\right)$ are

$$
\begin{aligned}
& \mathbf{R}_{k 0}\left(r, \omega_{k}\right)=\tilde{B}_{0}\left(r, \varepsilon, \omega_{k}\right) \cdot(1, \quad 0)^{T}=\left(b_{11}^{0}, \quad b_{21}^{0}\right)^{T}, \\
& \mathbf{R}_{k i}\left(r, \omega_{k}\right)=\tilde{B}_{i}\left(r, r_{i-1}, \omega_{k}\right) \cdot \tilde{B}\left(r_{i-1}, r_{0}, \omega_{k}\right) \cdot \tilde{B}_{0}\left(r_{0}, \varepsilon, \omega_{k}\right) \cdot(1, \quad 0)^{T}= \\
& =\tilde{B}_{i}\left(r, r_{i-1}, \omega_{k}\right) \cdot \tilde{B}\left(r_{i-1}, r_{0}, \omega_{k}\right) \cdot\left(b_{11}^{0}, \quad b_{21}^{0}\right)^{T} .
\end{aligned}
$$

Decomposition by eigenfunctions. Let $g(r)$ be piecewise continuous function having different analytic expressions on intervals $\left[\varepsilon, r_{0}\right)$ and $\left[r_{i-1}, r_{i}\right)$, i. e.,

$$
g(r)=\sum_{i=0}^{n} g_{i} \theta_{i}
$$


Function $g(r)$ expansion into Fourier series by eigenfunctions $R_{k}\left(r, \omega_{k}\right)$ of problem (19), (20) is as follows [12]

$$
g(r)=\sum_{k=1}^{\infty} g_{k} \cdot R_{k}\left(r, \omega_{k}\right)
$$

where Fourier coefficients $g_{k}$ in expansion (28) are calculated by the formula

$$
g_{k}=\frac{1}{\left\|R_{k}\right\|^{2}} \int_{\varepsilon}^{r_{n}} c \rho g(r) R_{k}\left(r, \omega_{k}\right) r^{2} d r
$$

It should be noted that $\left\|R_{k}\right\|^{2}$ is the norm square of eigenfunctions $R_{k}\left(r, \omega_{k}\right)$

$$
\left\|R_{k}\right\|^{2}=\int_{\varepsilon}^{r_{n}} c \rho r^{2} R_{k}^{2}\left(r, \omega_{k}\right) d r
$$

Construction of mixed problem solution for functions $v(r, \tau)$. The scheme of this problem solution construction by eigenfunctions method is described in papers $[6-7,11-13]$ in details. This solution can be presented in the following way:

$$
\begin{gathered}
v(r, \tau)=\sum_{k=1}^{\infty}\left[f_{k} \cdot e^{-\omega_{k} \tau}-\int_{0}^{\tau} e^{-\omega_{k}(\tau-s)} \gamma_{k}(s) d s\right] \cdot R_{k}\left(r, \omega_{k}\right)=\sum_{i=0}^{n-1} v_{i}(r, \tau) \cdot \theta_{i}, \\
v_{i}(r, \tau)=\sum_{k=1}^{\infty}\left[f_{k} \cdot e^{-\omega_{k} \tau}-\int_{0}^{\tau} e^{-\omega_{k}(\tau-s)} \gamma_{k}(s) d s\right] \cdot R_{k i}\left(r, \omega_{k}\right),
\end{gathered}
$$

where $f_{k}$ and $\gamma_{k}$ are expansion coefficients for initial condition and function relatively to Fourier system according to eigenfunctions system $R_{k}\left(r, \omega_{k}\right)$.

Boundary transition at $\varepsilon \rightarrow 0$ and description of original problem solution (1)-(4). On the basis of reduction method the original problem solution is presented as

$$
\begin{gathered}
T(r, \tau)=\lim _{\varepsilon \rightarrow 0}(u(r, \tau)+v(r, \tau))= \\
=\lim _{\varepsilon \rightarrow 0}\left(\psi(\tau)+\sum_{i=0}^{n-1} \sum_{k=1}^{\infty}\left[f_{k i} \cdot e^{-\omega_{k} \tau}-\int_{0}^{\tau} e^{-\omega_{k}(\tau-s)} \gamma_{k i}(s) d s\right] \cdot R_{k i}\left(r, \omega_{k}\right) \theta_{i}\right) .
\end{gathered}
$$

Note! The characteristic equation (26) of the eigenvalues problem (19), (20) after the boundary transition is obviously as follows

$$
\left(\alpha r_{n}^{2} c_{11}+c_{21}\right) \cdot \frac{\sin \left(\beta_{0}\left(r_{0}\right)\right)}{\beta_{0} r_{0}}+\left(\alpha r_{n}^{2} c_{12}+c_{22}\right) \cdot \frac{\beta_{0} r_{0} \cos \left(\beta_{0} \cdot r_{0}\right)-\sin \left(\beta_{0} r_{0}\right)}{\beta_{0}} \cdot \lambda_{0}=0,
$$


and the corresponding eigenvectors (27) are depicted as follows:

$$
\begin{aligned}
& \mathbf{R}_{k 0}\left(r, \omega_{k}\right)=\tilde{B}_{0}(r, 0, \omega) \cdot(1, \quad 0)^{T}=\left(\frac{\sin \left(\beta_{0} r\right)}{\beta_{0} r}, \quad \frac{\beta_{0} r \cos \left(\beta_{0} \cdot r\right)-\sin \left(\beta_{0} r\right)}{\beta_{0}} \lambda_{0}\right)^{T}, \\
& \mathbf{R}_{k 0}\left(0, \omega_{k}\right)=\left(\begin{array}{ll}
1, & 0
\end{array}\right)^{T} \\
& \mathbf{R}_{k i}\left(r, \omega_{k}\right)=\tilde{B}_{i}\left(r, r_{i-1}, \omega_{k}\right) \cdot \tilde{B}\left(r_{i-1}, r_{0}, \omega_{k}\right) \cdot\left(b_{11}^{0}, \quad b_{21}^{0}\right)^{T}= \\
& =\tilde{B}_{i}\left(r, r_{i-1}, \omega_{k}\right) \cdot \tilde{B}\left(r_{i-1}, r_{0}, \omega_{k}\right) \cdot\left(\frac{\sin \left(\beta_{0} r_{0}\right)}{\beta_{0} r_{0}}, \quad \frac{\beta_{0} r_{0} \cos \left(\beta_{0} \cdot r_{0}\right)-\sin \left(\beta_{0} r_{0}\right)}{\beta_{0}} \lambda_{0}\right)^{T} .
\end{aligned}
$$

Model example. We consider the system of seven-layer isotropic spherical spheres requiring the determination of non-stationary temperature field distribution as the model numerical example. The heat engineering characteristics of the materials the spheres are made

\begin{tabular}{|c|c|c|c|c|c|c|c|}
\hline Parameter & Layer 1 & Layer 2 & Layer 3 & Layer 4 & Layer 5 & Layer 6 & Layer 7 \\
\hline Radius $r$,ì & $0-0,05$ & $\begin{array}{c}0,05- \\
0,08\end{array}$ & $\begin{array}{c}0,08- \\
0,15\end{array}$ & $\begin{array}{c}0,15- \\
0,25\end{array}$ & $\begin{array}{c}0,25- \\
0,33\end{array}$ & $\begin{array}{c}0,33- \\
0,39\end{array}$ & $0,39-0,43$ \\
\hline $\begin{array}{l}\text { Coefficient of thermal } \\
\text { conductivity } \lambda, \mathrm{W} / \mathrm{m} \cdot{ }^{0} \mathrm{C}\end{array}$ & 0,93 & 209 & 64 & 34,6 & 2,93 & 58 & 1,55 \\
\hline $\begin{array}{l}\text { Specific mass heat capacity } \\
\qquad c, \mathrm{Jg} \cdot{ }^{\circ} \mathrm{C}\end{array}$ & 837 & 894 & 389 & 130 & 921 & 470 & 770 \\
\hline Density $\rho, \mathrm{kg} / \mathrm{m}^{3}$ & 2000 & 2680 & 8000 & 11400 & 2800 & 7800 & 2200 \\
\hline Initial temperature $t(r, 0),{ }^{0} \mathrm{C}$ & 20 & 20 & 20 & 20 & 20 & 20 & 20 \\
\hline $\begin{array}{c}\text { Law of environment temperature } \\
\text { change, } \psi(\tau),{ }^{0} \mathrm{C}\end{array}$ & \multicolumn{7}{|c|}{$\psi(\tau)=345 \lg \left(\frac{8 \tau}{60}+1\right)+20$} \\
\hline $\begin{array}{c}\text { Coefficient of heat exchange } \\
\text { with the environment, } \\
\alpha \alpha, \mathrm{W}^{2} \cdot{ }^{\circ} \mathrm{C}\end{array}$ & \multicolumn{7}{|c|}{25} \\
\hline
\end{tabular}
of and the initial data for calculation are given in Table 1.

Table 1

Heat-technical property of the materials

Using the proposed method for calculating the non-stationary temperature field distribution in the system of multilayer spherical spheres and Maple 13 software [13], we derive the solution of the given problem as Table 2. It should be noted that in order to get the result with the given accuracy, the first 50 roots of characteristic equation were used. 
Table 2

Distribution of the temperature field in the system of multilayered spherical bullets, ${ }^{0} \mathrm{C}$

\begin{tabular}{|c|c|c|c|c|c|c|c|c|c|}
\hline \multirow{2}{*}{ time } & \multicolumn{9}{|c|}{ Layer coordinates IIapy, m } \\
\cline { 2 - 10 } & $\mathbf{0}$ & $\mathbf{0 , 0 5}$ & $\mathbf{0 , 1}$ & $\mathbf{0 , 1 5}$ & $\mathbf{0 , 2}$ & $\mathbf{0 , 2 5}$ & $\mathbf{0 , 3}$ & $\mathbf{0 , 3 5}$ & $\mathbf{0 , 4 3}$ \\
\hline 0 min. & 20 & 20 & 20 & 20 & 20 & 20 & 20 & 20 & 20 \\
\hline 1 min. & 20 & 20 & 20 & 20 & 20 & 20 & 20 & 20 & 52,66 \\
\hline 3 min. & 20 & 20 & 20 & 20 & 20 & 20 & 20 & 20,02 & 102,2 \\
\hline 10 min. & 20 & 20 & 20 & 20 & 20 & 20 & 20,38 & 24,12 & 210,9 \\
\hline 20 min. & 20 & 20,03 & 20,04 & 20,06 & 20,13 & 20,24 & 25,52 & 41,20 & 299 \\
\hline 30 min. & 20,06 & 20,54 & 20,59 & 20,76 & 21,19 & 21,71 & 36,63 & 64 & 353,3 \\
\hline 45 min. & 21,22 & 24,04 & 24,22 & 24,83 & 26,24 & 27,81 & 59,79 & 100,9 & 409,6 \\
\hline 1 hour & 25,68 & 32,18 & 32,52 & 33,63 & 36,14 & 38,81 & 86,56 & 137,7 & 453,3 \\
\hline 2 hours & 85,32 & 103,6 & 104,3 & 106,7 & 112,1 & 117,5 & 201,9 & 272,6 & 582,2 \\
\hline 3 hours & 183,4 & 205,6 & 206,5 & 209,3 & 215,4 & 221,7 & 315,4 & 389,5 & 678,5 \\
\hline
\end{tabular}

It should be noted that the set problem is the model aimed to illustrate the possibilities of the proposed method.

Conclusions. The direct method was used to solve the oroginal problem, moreover, the idea of the boundary transition was used for the first time. In general statement (the function $\psi(\tau)$ is supposed to be arbitrary), this problem was solved for the first time. In the case of the system of two enclosed spheres and at $\psi(\tau) \equiv 0$, the obtained solution is the same as the known one [3]. The generalization of the obtained results for cylindrical coordinate system is merely technical problem but not fundamental one. Since the general scheme of the investigation of temperature fields distribution in multilayer structures with the arbitrary number of layers in the presence of internal heat sources is studied in details, then setting and solving of such problems does not cause any difficulties.

\section{References}

1. Singh, Suneet, and Prashant K. Jain. "Analytical solution for three-dimensional, unsteady heat conduction in a multilayer sphere." Journal of Heat Transfer 138.10 (2016): 101301. https://doi.org/10.1115/ 1.4033536.

2. De Monte, Filippo. "Unsteady heat conduction in two-dimensional two slab-shaped regions. Exact closedform solution and results." International Journal of Heat and Mass Transfer 46.8 (2003): 1455-1469. https://doi.org/10.1016/S0017-9310(02)00417-9

3. Lykov, A. V. (1967) Teoriia teploprovodnosti, Vysshaia shkola, Mockow, USSS. [In Russian].

4. Özişik M. N., Orlande H. R. B., Colaço M. J., Cotta R. M. Finite Difference Methods in Heat Transfer, Second Edition. New York: CRC Press, 2017. 580 p.

5. Gosz M. R. Finite Element Method: Applications in Solids, Structures, and Heat Transfer. New York: CRC Press, 2017. 400 p. https://doi.org/10.1201/9781315275857 
6. O. Y. Pazen and R. M. Tatsii. "Direct (classical) method of calculation of the temperature field in a hollow multilayer cylinder". Journal of Engineering Physics and Thermophysics, vol. 91, no. 6, pp. 1373-1384, November 2018. https://doi.org/10.1007/s10891-018-1871-3

7. Tatsii R. M., Stasiuk M. F. and Pazen O. Y. "Pryamoy metod rascheta temperaturnogo polya v mnogosloynoy poloy sfericheskoy konstruktsii" Vestnik Kokshetauskogo tekhnicheskogo instituta, no. 1 (29), pp. 9-20, 2018. (In Russian).

8. Pazen O. Y. and Tatsii R. M., General boundary-value problems for the heat conduction equation with piecewise-continuous coefficients, Journal of Engineering Physics and Thermophysics, vol. 89, no. 2, pp. 357-368, March 2016. https://doi.org/10.1007/s10891-016-1386-8.

9. Arsenin, V. Ya. (1974) Metody matematicheskoii fizyky, Nauka, Moscow, USSR. [In Russian].

10. Tihonov A. N. and Samarskii A. A. (1977) Uravnenie matematicheskoii fizyky, Nauka, Moscow, USSR. [In Russian].

11. Tatsiy R. M., Pazen O. Yu. "Pryamyy metod rozrakhunku nestatsionarnoho temperaturnoho polya za umov pozhezhi”, Pozhezhna bezpeka: zb. nauk. pr. Lviv: LDU BZHD, 2015. № 26. S. 135-141. [In Ukrainian].

12. Tatsiy R. M., Ushak T. I., Pazen O. Yu. Zahalna tretya krayova zadacha dlya rivnyannya teploprovidnosti z kuskovo-stalymy koefitsiyentamy ta vnutrishnimy dzherelamy tepla, Pozhezhna bezpeka: Zb. nauk. pr. Lviv: LDU BZHD, 2015. № 27. S. 120-126. [In Ukrainian].

13. Pazen O. Y. "Mathematical modelling and computer simulation of direct method for studying boundary value problem of thermal conductivity" Problems of Infocommunications. Science and Technology, pp. 73-76. 2017. https://doi.org/10.1109/INFOCOMMST.2017.8246353

\section{Список використаної літератури}

1. Singh, Suneet, and Prashant K. Jain. Analytical solution for three-dimensional, unsteady heat conduction in a multilayer sphere. Journal of Heat Transfer. 138.10 (2016): 101301. https://doi.org/10.1115/ 1.4033536.

2. De Monte, Filippo. Unsteady heat conduction in two-dimensional two slab-shaped regions. Exact closedform solution and results. International Journal of Heat and Mass Transfer 46.8 (2003): 1455-1469. https://doi.org/10.1016/S0017-9310(02)00417-9

3. Лыков А. В. Теория теплопроводности. М.: Высшая школа, 1967. 600 с.

4. Özişik M. N., Orlande H. R. B., Colaço M. J., Cotta R. M. Finite Difference Methods in Heat Transfer, Second Edition. New York: CRC Press, 2017. 580 p.

5. Gosz M. R. Finite Element Method: Applications in Solids, Structures, and Heat Transfer. New York: CRC Press, 2017. 400 p. https://doi.org/10.1201/9781315275857

6. Pazen O. Y. and Tatsii R. M. Direct (classical) method of calculation of the temperature field in a hollow multilayer cylinder. Journal of Engineering Physics and Thermophysics. Vol. 91. No. 6. Pp. 1373-1384. November 2018. https://doi.org/10.1007/s10891-018-1871-3

7. Тацій Р. М., Стасюк М. Ф., Пазен О. Ю. Прямой метод расчета температурного поля в многослойной полой сферической конструкции. Вестник Кокшетаутского технического института. 2018. № 1 (29). C. 9-20.

8. Pazen O. Y. and Tatsii R. M. General boundary-value problems for the heat conduction equation with piecewise-continuous coefficients. Journal of Engineering Physics and Thermophysics. Vol. 89. No. 2. Pp. 357-368. March 2016. https://doi.org/10.1007/s10891-016-1386-8.

9. Арсенин В. Я. Методы математической физики. М.: Наука, 1974. 432 с.

10. Тихонов А. Н., Самарский А. А. Уравнения математической физики. М.: Наука, 1977. 735 с.

11. Тацій Р. М., Пазен О. Ю. Прямий метод розрахунку нестаціонарного температурного поля за умов пожежі. Пожежна безпека. Зб. наук. пр. Львів: ЛДУ БЖД. 2015. № 26. С. 135-141.

12. Тацій Р. М., Ушак Т. І., Пазен О. Ю. Загальна третя крайова задача для рівняння теплопровідності 3 кусково-сталими коефіцієнтами та внутрішніми джерелами тепла. Пожежна безпека. Зб. наук. пр. Львів: ЛДУ БЖД. 2015. № 27. С. 120-126.

13. Pazen O. Y. and Tatsii R. M. General boundary-value problems for the heat conduction equation with piecewise-continuous coefficients. Journal of Engineering Physics and Thermophysics. Vol. 89. No. 2. Pp. 357-368. March 2016. https://doi.org/10.1109/INFOCOMMST.2017.8246353 


\title{
УДК 614.841.34
}

\section{ПРЯМИЙ МЕТОД ДОСЛІДЖЕННЯ ТЕМПЕРАТУРНОГО ПОЛЯ У СИСТЕМІ БАГАТОШАРОВИХ СФЕРИЧНИХ ТІЛ}

\author{
Роман Тацій; Олег Пазен
}

\author{
Львівський державний університет безпеки життєдіяльності, \\ Львів, Украӥна
}

Резюме. Запропонована робота присвячена застосуванню прямого методу до дослідження процесів теплообміну в системі - куля всередині багатомарової сферичної оболонки (конструкції). Припускається, що між шарами иієї системи існує ідеальний тепловий контакт, а теплообмін між навколишнім середовищем та зовнішньою поверхнею відбувається за законом Ньютона-Ріхмана. Тобто виконуються умови третього роду. Закон зміни температури навколишнього середовища є довільною функиією часу та рівномірно розподілений у приповерхневому шарі так, щзо ізотерми всередині конструкиії являють собою концентричні сфери. Отож задача є симетричною $і$ в такій постановиі розв'язана вперше. Для розв'язування такої задачі паралельно ставиться допоміжна задача про визначення розподілу нестаціонарного температурного поля у багатошаровій порожнистій сферичній конструкиії з «вилученою» кулею достатньо малого радіуса. При иььму умова симетрії вихідної задачі замінюється умовою другого роду на внутрішній поверхні иієї конструкиії. Реалізація розв'язку допоміжної задачі проводиться шляхом застосування методу редукції з використанням концепції квазіпохіднх. Надалі використовується схема Фур'є із застосуванням модифікованого методу власних функиій. Для знаходження розв'язку вихідної задачі використано ідею граничного переходу иляхом прямування радіуса вилученої кулі до нуля. Встановлено, щзо при такому підході всі власні функції відповідної задачі на власні значення не мають особливостей в нулі, а це означає, щзо й розв'язки вихідної задачі є обмеженими у всій конструкиії. Для ілюстрації запропонованого методу розв'язано модельний приклад про знаходження розподілу температурного поля у системі семишарових сферичних конструкцій з різними теплофізичними характеристиками матеріалів. Результати обчислень представлені у вигляді таблиці про зміну температури залежно від часу та просторової координати. Узагальнення отриманих результатів для випадку інших крайових умов (наприклад, першого роду) будь-якої скінченної кількості багатошарової сферичної конструкиї не викликає жодних труднощңів.

Ключові слова: багатошарова куля, квазіпохідна, прямий метод, граничний перехід. 